$$
\text { "olahgal" — 2007/2/16 — 16:31 — page 307 — \#1 }
$$

\title{
Die aus der Studienzeit stammenden Aufzeichnungen des Johann Bolyai über die Würfelverdoppelung
}

\author{
RÓBERT OlÁH-GÁL
}

\begin{abstract}
Hereinafter we are going to show that Bolyai Janos was preoccupied by the problem of the Duplication of the Cube, which was unknown until now by the rich Bolyai-literature.

This problem was solved using the Parabola, the Hyperbola and the Cissoide already in the ancient times. The Cissoide was created by Diocles especially for the constuction of the Duplication of the Cube without Compass and Straightedge. The hereinafter "deciphered" document of Bolyai was written during his university studies. In his study he presents the solutions discovered by then and tries to give a new one. We transcribed his notations to the present-day use and complemented it where it was necessary.

The mathematics historically background and the explication is very detailed described by Van der Waerden in Erwachende Wissenschaft [7], which is to find on English, German and Hungarian, too. That's because we dispense with this [8].
\end{abstract}

Key words and phrases: Johann Bolyai, cube duplication.

ZDM Subject Classification: 01A20, 01A55, 51-03, 51N20.

Es ist eine Tatsache der Mathematikgeschichte, dass die deutschen, mit gothischen Buchstaben geschriebenen Aufzeichnungen von Johann Bolyai über die Winkeldreiteilung erst von Paul Stäckel im Jahre 1898, zwischen den in Neumarkt aufbewahrten Handschriften gefunden worden sind. Diese veröffentlichte er 1913 in deutscher Sprache, 1914 erschienen sie im ersten Band der zweibändigen, bis heute der gründlichsten Bolyai-Monographie [4]. Diese Aufzeichnungen wurden

Copyright (c) 2006 by University of Debrecen 
auch von Gyula Szőkefalvi-Nagy auf einer klaren und verständlichen Weise vorgestellt [5]. Aber die Bolyai - Forscher haben nicht bemerkt, dass sich neben diesem Aufzeichnungsteil auch die Ausführung eines anderen Problems des Altertums befindet, und zwar das der Würfelverdoppelungen [2], [3].

Einer griechischen Sage nach ist auf der Insel Delos eine Epidemie ausgebrochen (wahrscheinlich die Pest). Die Götter haben von den Einwohnern gefordert, dass, wenn sie ihren würfelformigen Altar mit einem anderen Würfelaltar mit genau zweimal so großem Rauminhalt austauschen, wird die Krankheit verschwinden. (Die Würfelverdoppelung ist auch als das Problem von Delos bekannt).

Wenn die Seiten des originellen Würfels einheitlich gewählt sind, besteht das Problem von Delos aus der Lösung der Gleichung $x^{3}-2=0$. Die Wurzel der Gleichung ist mit Hilfe des Zirkels und des Lineals nicht konstruierbar (mit anderen Kurven lösten es mehrere schon im Altertum). Für die Konstruktion der Verdoppelung des Würfels schuf Diokles den als Zissoide genannten geometrischen Ort (Kurve). Zur Konstruktion der Zissoide zeichnen wir den Kreis mit dem Durchmesser $a$. Wir betrachten den Endpunkt O des Durchmessers als Zentrum einer Radiusreihe in der Ebene; durch den anderen Endpunkt ziehen wir die Tangente zum Kreis. Wenn ein ausgewählter Radius den Kreis im Punkt $B$ die Tangente in $C$ durchquert, dann ist $P$ der Punkt der Zissoide, die auf dem Strahl $O B$ liegt und für die gilt: $O P=B C$.

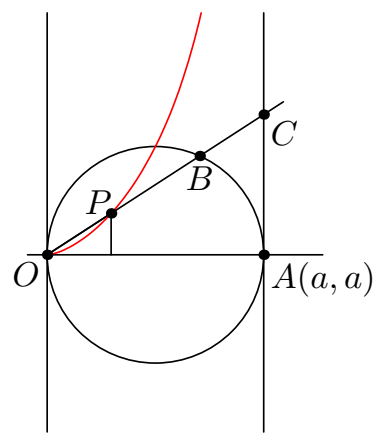

Abbildung 1

Die Lösung des Problems der Würfelverdoppelung mit Hilfe der Parabel, der Hyperbel und der Zissoide war schon im Altertum bekannt. Die Zissoide war von Diokles wegen der Konstruktion der Würfelverdoppelung ohne Zirkel und Lineal geschafft. 


$$
\text { "olahgal" — 2007/2/16 — 16:31 — page 309 — \#3 }
$$

$\underline{\text { Die aus der Studienzeit stammenden Aufzeichn. des J. Bolyai ü. die Würfelverdoppelung } 309}$

Eigentlich haben schon die Griechen erkannt, dass das Problem der Würfelverdoppelung gleich mit dem n-maligen Vervielfachen des Würfels ist (d.h. die Konstruktion eines Würfels mit genau $n$-mal so großem Rauminhalt wie der des ursprünglichen). Johann Bolyai beginnt mit der Lösung des $n$-maligen. Aber bei der letzten Konstruktion sieht er ein, dass es nur für $n=2$ gilt. Vielleicht ist das die Letzte, die original ist und bis dann unbekannt war. Wir haben die Bezeichnung von Bolyai auf den heutigen Gebrauch umgeschrieben, und wo es nötig war, haben wir es ergänzt.

Der mathematikhistorische Hintergrund und die Erklärung der Würfelverdoppelung ist sehr ausführlich von Van der Waerden beschrieben, in Erwachende Wissenschaft [7], was es auch auf Englisch, Deutsch und Ungarisch gibt. Deshalb sehen wir davon ab.

Die Konstruktion mit Hilfe der Zissoide (wie sie sich in der Handschrift von Johann Bolyai befindet [1]).

Aufgabe: Es sei ein Würfel mit der Seite $a$ gegeben, ein anderer Würfel mit der Seite $x$ wird gesucht, so dass $x^{3}=n a^{3}$.

Die Methode der Lösung: man suche eine mittlere geometrische Proportion zwischen $a$ und $n a$, und das ist: $a: x=x: y=y: n a$, so $y=x^{2} / a$, oder $y^{2}=x^{4} / a^{2}, x^{4} / a^{2}=n a x$, weil $x^{3}=n a^{3}$.

Es sei $a=O K$, und $n a=O D$ gegeben. Zeichnen wir einen Kreis mit dem Durchmesser $D K$. Die Rechtgerade in $O$ durchquert den Kreis in Punkt $B$. Es sei $b=O B$. Dann $b^{2}=n a^{2}$. Es sei ein anderer Kreis mit dem Zentrum $O$ und dem Radius $b$. Es sei in Punkt $B$ die Radiusreihe $B C, B N, B P$, und konstruiren wir eine Zissoide in Punkt $B$.

$B N \cap \operatorname{Kreis}(O, b)=Q, B P \cap \operatorname{Kreis}(O, b)=A . B C=2 b$.

Auf Grund der Ähnlichkeit können wir hinzufügen, dass

$$
\frac{B S}{S L}=\frac{B O}{O M}\left(=\frac{b}{x}\right) \Rightarrow S L=\frac{B S \cdot x}{b} .
$$

Genauso

$$
\frac{C S}{S L}=\frac{C O}{O K}\left(=\frac{b}{a}\right) \Rightarrow S L=\frac{a \cdot C S}{b} .
$$

Also, $B S \cdot x=C S \cdot a$ und die beiden Seiten $C S$-mal genommen, erhalten wir im $\triangle B Q C$ auf Grund des Höhensatzes.

$B S \cdot C S \cdot x=a \cdot C S^{2}=R Q^{2} \cdot x, R Q^{2}=B R \cdot R C=B S \cdot C S$, aber

$$
\frac{B R}{R Q}=\frac{B O}{O M}\left(=\frac{b}{x}\right)
$$




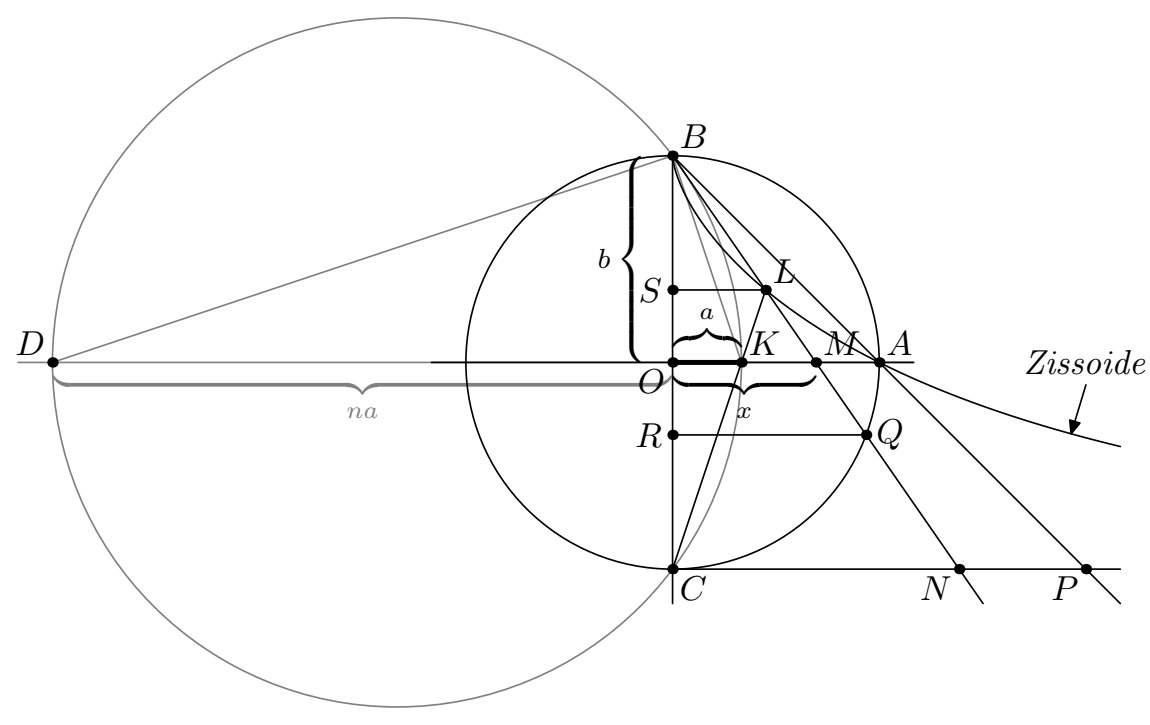

Abbildung 2

von hier

wovon

$$
R Q^{2}=\frac{x^{2} \cdot B R^{2}}{b^{2}}
$$

$$
a \cdot C S^{2}=\frac{x^{2} \cdot B R^{2}}{b^{2}} x
$$

weil $C S=B R, x^{3}=a^{3} n$.

Johann Bolyai hat das nur als Einleitung bestimmt, danach hat er kurz die Lösungen gegeben, die erste, die zweite und die dritte.

\section{Lösung}

Zeichnen wir das Rechteck $O A B C$, mit der Höhe $O C=a$ und der Breite $O A=n a$.

Dann zeichen wir den Kreis um das Rechteck herum. Nehmen wir die Gerade $O C$ als Achse $y$, und die Gerade $O A$ als Achse $x$, dann zeichnen wir eine gleichseitige Hyperbel, die durch den Punkt $B$ geht.

Diese Hyperbel durchquert den Kreis auch im Punkt $D$. Die Gerade $B D$ durchquert die Achse $x$ im Punkt $F$, die Achse $y$ im Punkt $G$. 


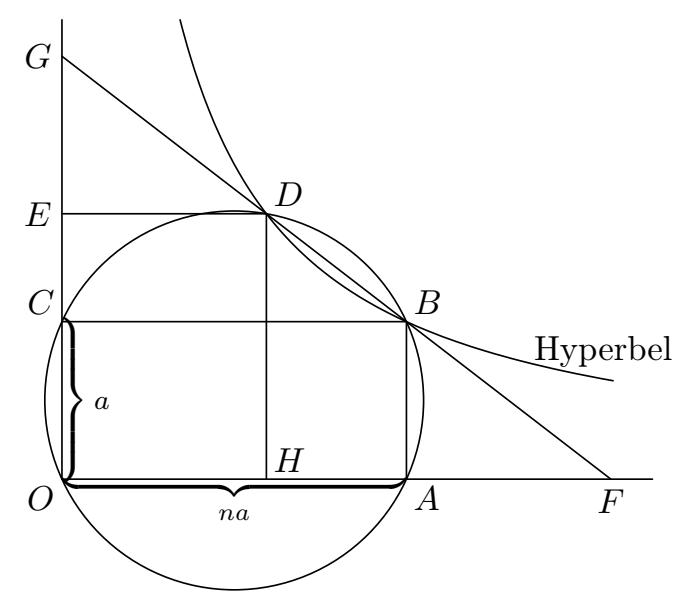

Abbildung 3

Die Koordinaten des Punktes $D$ sind $O H$ und $H D=O E$, deshalb $x y=$ $n a \cdot a=O A \cdot A B=O H \cdot H D$ (im Allgemeinen $x y=\alpha \beta$, oder rechtwinklige Hyperbel, die Assymptoten der Hyperbel sind die Koordinatenachsen, also $O A$ ist die $x$-Achse und $O C$ die $y$-Achse).

Weil die Hyperbel gleichseitig ist, ist $D G=B F$ und $B G=D F$. Daraus folgt, dass $G E=B A=a$ und $C B=H F=n \cdot a$.

Die Dreiecke $H F D$ und $C B G$ sind kongruent. Offenbar ist $O D$ senkrecht auf $G F$; weil $C O D \angle=O D H \angle=D B C \angle$ (Umkreiswinkel), und deshalb $E D^{2}=$ $G E \cdot E O$ (der Höhensatz im $O D G \triangle$ ).

$E D: E O=O H: D H$.

So $E D: E O=G E: E D=O H: D H$ oder $G E: E D=E D: E O=$ $O H: D H=D H: H F$ und so $a: E D=E D: D H=D H: H F$. Also das gesuchte $x$ ist die Strecke $E D$.

\section{Lösung}

Es sei $p$ der Parameter der Parabel $y^{2}=p x$ und $q$ der Parameter der Parabel $x^{2}=q y$, daraus folgt, dass $p: y=y: x=x: q$. Wir nehmen $q$ als $q=n p$ und so ist $y$ die gesuchte Seite.

Ausführung: Es sei der Würfel mit der Seite $p$ gegeben! 


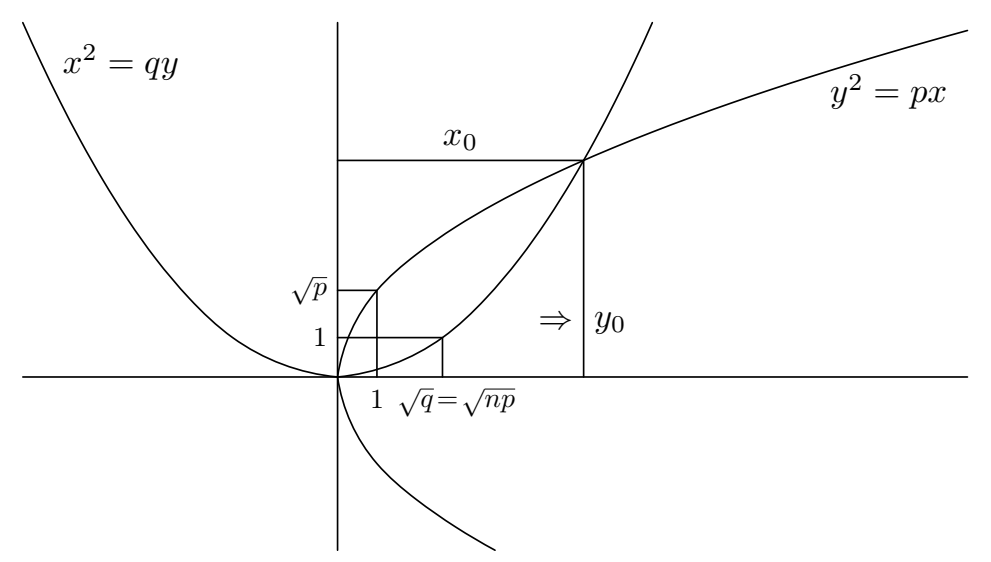

Abbildung 4

Der Parameter $q$ ist so gewählt dass $q=n p$.

$$
\left\{\begin{array}{l}
x_{0}^{2}=n p y_{0} \\
y_{0}^{2}=p x_{0},
\end{array}\right.
$$

für den Schneidepunkt der beiden Parabel $\Rightarrow y_{0}^{4}=p^{2} x_{0}^{2}=n p^{3} y_{0}, \Rightarrow y_{0}^{3}=n p^{3}$.

Dann gilt es schon für den Schneidepunkt der beiden Parabeln, dass:

$$
\frac{p}{y_{0}}=\frac{y_{0}}{x_{0}}=\frac{x_{0}}{q} \text {. }
$$

Also die Seite des gesuchten Würfels mit (zweimal) n-mal so großem Rauminhalt ist genau $y_{0}$.

\section{Lösung}

Diese Konstruktion gilt nur, wenn $n=2$; das wurde später von Johann Bolyai hinzugefügt!

Es sei $A B=n a$. Halbieren wir die $A B$-Strecke mit dem Punkt $C$. Die halbierende Senkrechte der $A C$ sei $O D$ so, dass $O D=A C$. Zeichnen wir einen Kreis mit dem Mittelpunkt $O$, und dem Radius $A O$. Nehmen wir $A B$ als die Achse $x$ des Koordinatensystems, und zeichnen wir die Parabel mit der Länge der $A C$ Strecke als Parameter. $y^{2}=A C \cdot x=(n a / 2) x$. Es sei der Schneidepunkt des Kreises und der Parabel $E$. Bezeichnen wir den Grundpunkt der Senkrechte aus $A$ auf $A B$ mit $H$, sie schneidet den Kreis noch im Punkt $F$. Es sei $G$ der Grundpunkt der Senkrechte aus $O$ auf $E F$. Weil $C E F \angle=F A C \angle$ (Umkreiswinkeln), 


$$
\text { "olahgal" — 2007/2/16 — 16:31 — page 313 — \#7 }
$$

$\underline{\text { Die aus der Studienzeit stammenden Aufzeichn. des J. Bolyai ü. die Würfelverdoppelung } 313}$

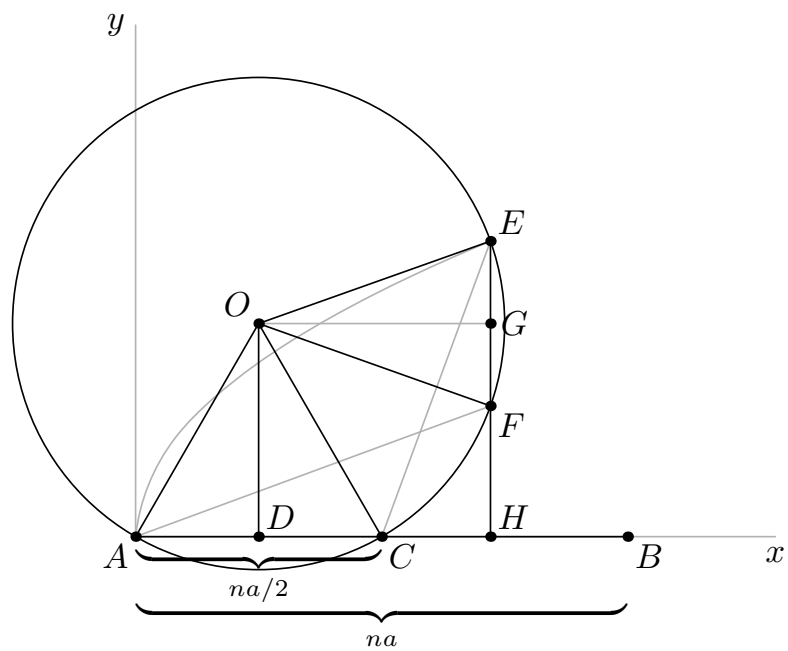

Abbildung 5

das $\triangle A H F$ ist, ähnlich zu dem Dreieck $E H C$. Deshalb $A H: H E=H F: H C$, auf der anderen Seite $H C=A H-A C$ so $A H^{2}-A H \cdot A C=H E \cdot H F$.

Aber wegen der Parabel $H E^{2}=A C \cdot A H$, so $H E \cdot H F+H E^{2}=A H^{2}=$ $H E(H F+H E)$.

aber $2 \cdot H G=H G+G F+F H=H G+E G+H F=E H+F H=A B$ (wir sind davon ausgegangen, dass $E G=G F$ und $G H=D O=A C=n a / 2)$

Also aus $A H^{2}=H E \cdot A B$, folgt $E H: A H=A H: A B$ also $A C: E H=$ $E H$ : $A H$, daraus folgt, dass die gesuchte Würfelseite $x$ gleich $E H$ ist!

Auf Grund der mathematikgeschichtlichen Forschungen können wir feststellen, dass von diesen drei Lösungen der Würfelverdoppelung vielleicht nur die letzte der Fachliteratur nicht bekannt ist (also die, die nur für $n=2$ gilt). Aber auch die von Stäckel, Szőkefalvi-Nagy vorgestellte Winkeldreiteilung war schon in der mathematischen "Literatur" bekannt. Diese Konstruktionen haben viel mehr eine didaktische Bedeutung, sie können zum Beispiel in Gymnasien vorgestellt werden; man kann solche Konstruktionen fordern. Aber in der ungarischen Mathematikgeschichte war Johann Bolyai der erste, der alle 3 griechischen Probleme gelöst hat, aber nur mit der Bemerkung, dass er für die Würfelverdoppelung die Parabel, für die Winkeldreiteilung die Hyperbel benutzt hat und die Quadratur des Kreises im Rahmen seiner nicht euklidischen Geometrie gelöst hat. 


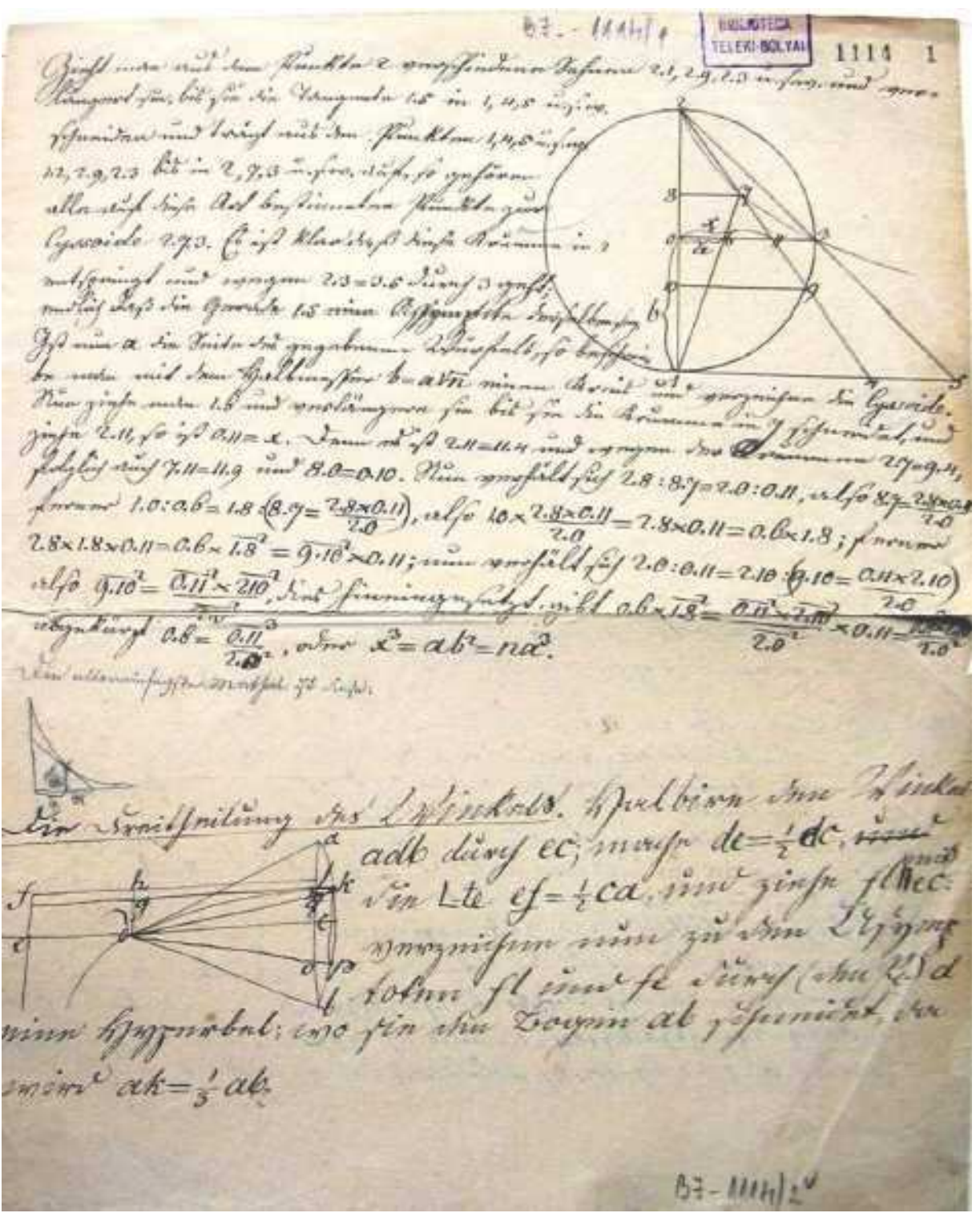

Abbildung 6 


$$
\text { "olahgal" — 2007/2/16 — 16:31 — page 315 — \#9 }
$$

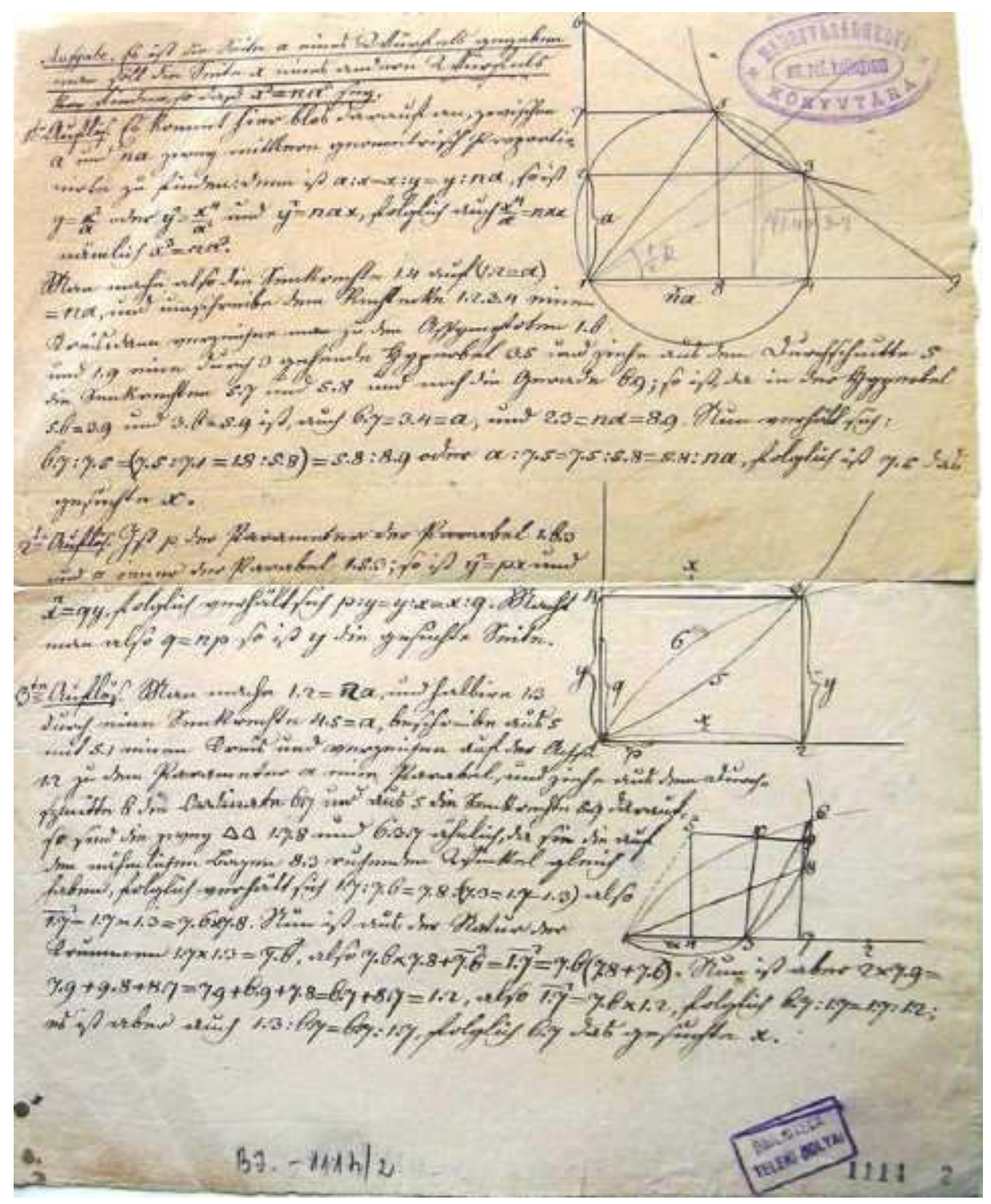

Abbildung 7 


$$
\text { "olahgal" — 2007/2/16 — 16:31 — page } 316 \text { — \#10 }
$$

316 R. O-G. : Die aus der Stud.zeit stamm. Aufzeichn. des J. Bolyai ü. d. Würfelverdoppelung

\section{Literatur}

[1] Teleki-téka, Marosvásárhely, Bolyai János kéziratai. BJ-1114/2, 1114/2v .

[2] Weszely Tibor, Bolyai János matematikai munkássága, Kriterion Könyvkiadó, Bukarest, 1981, 75-77.

[3] Tóth Sándor, A szögharmadolás problémája és Bolyai Jánosnak erre vonatkozó diákkori eredménye, Mat. és Fiz. Lapok 1, Kolozsvár (Cluj) (1960).

[4] Paul Stäckel, Wolfgang und Johann Bolyai geometrische Untersuchungen: mit Unterstützung der Ungarischen Akademie der Wissenschaften, in: Urkunden zur Geschichte der nichteuklidischen Geometrie; 2., (Paul Stäckel, ed.), B. G. Teubner, Leipzig, Berlin, 1913.

[5] Szőkefalvi-Nagy Gyula, Bolyai János szögharmadolása, Matematikai Lapok 4 (1953), 84-86.

[6] Oláh-Gál Róbert, Horváth Sándor, Deep geometrical thoughts from some - until now not published - manuscripts of János Bolyai, History of Mathematics \& Teaching of Mathematics, University of Miskolc, May 21-23, 2004.

[7] Van der Waerden, Erwachende Wissenschaft, Birkhäuser, Basel/Stuttgart, 1966.

[8] http://www.cs.mcgill.ca/ cs507/projects/1998/zafiroff/.

BABES-BOLYAI UNIVERSITY

CLUJ-NAPOCA

STR. KOGALNICEANU NR.1.

ROMANIA

E-mail: olah_gal@topnet.ro

(Received January, 2006) 